\title{
Myocardial regeneration therapy for ischemic cardiomyopathy with cyclin A2
}

\author{
Y. Joseph Woo, MD, ${ }^{\text {a }}$ Corinna M. Panlilio, BA, ${ }^{\text {a }}$ Richard K. Cheng, MD, ${ }^{\text {b }}$ George P. Liao, MB, ${ }^{\text {a }}$ Erik E. Suarez, MD, \\ Pavan Atluri, MD, and Hina W. Chaudhry, MD ${ }^{\mathrm{b}}$
}

From the Division of Cardiothoracic Surgery, ${ }^{a}$ Department of Surgery, University of Pennsylvania School of Medicine, Philadelphia, Pennsylvania; and Division of Cardiology, ${ }^{\mathrm{b}}$ Department of Medicine, Columbia University School of Medicine, New York, New York.

This work was supported in part by grants from the NIH National Heart Lung and Blood Institute/Thoracic Surgery Foundation for Research and Education HL072812 (Y.J.W.); NIH National Heart Lung Blood Institute HL067048-03 (H.W.C.); NIH, National Heart Lung Blood Institute-Ruth L. Kirschstein National Research Service Award, Individual Fellowship 1 F32 HL79769-01 (P.A.); and AHA Heritage Affiliate Medical Student Research Fellowship (R.K.C.).

Hina Chaudhry reports a patent application for the use of cyclin A2 for cardiac repair.

Read at the Eighty-sixth Annual Meeting of The American Association for Thoracic Surgery, Philadelphia, Pa, April 29-May 3, 2006.

Received for publication April 28, 2006; revisions received June 20, 2006; accepted for publication July 7, 2006.

Address for reprints: Y. Joseph Woo, MD, Department of Surgery, University of Pennsylvania, Silverstein 6, 3400 Spruce St, Philadelphia, PA 19104 (E-mail: wooy@ uphs.upenn.edu).

J Thorac Cardiovasc Surg 2007;133:927-33

0022-5223/\$32.00

Copyright $(9) 2007$ by The American Association for Thoracic Surgery

doi:10.1016/j.jtcvs.2006.07.057
Objective: Heart failure therapies ranging from revascularization to remodeling to replacement are variably effective. Theoretically, endogenous repair via myocardial regeneration would be an ideal therapy. This study examined the ability to initiate regeneration by adenoviral-mediated expression of the cell cycle regulator cyclin A2. Our prior studies have demonstrated robust cyclin A2 transgene expression and marked antiphosphorylated histone $\mathrm{H} 3$ activity with this strategy, indicating the induction of cardiomyocyte mitosis.

Methods: Adult male, Lewis rats underwent left anterior descending coronary artery ligation followed by intramyocardial delivery of either cyclin A2 adenoviral vector $(n=8)$ or empty adeno-null vector as a control $(n=8)$ into the peri-infarct border zone. In vivo myocardial function was analyzed by echocardiography and invasive left ventricular pressure catheter at 6 weeks, when the animals are traditionally in heart failure. Hearts were explanted for immunoblotting and left ventricular geometric analysis. Cellular proliferation was assessed by proliferating cellular nuclear antigen expression.

Results: Cyclin A2 hearts exhibited improved left ventricular function as compared with controls including enhanced cardiac output ( $32 \pm 3.3$ vs $26 \pm 5.0$ $\mathrm{mL} / \mathrm{min}, P<.05)$, stroke volume $(0.16 \pm 0.04 \mathrm{vs} 0.11 \pm 0.04 \mathrm{~mL}, P<.05)$, ejection fraction $(72 \% \pm 7.4 \%$ vs $46 . \% \pm 8.5 \%, P<.05)$, fractional shortening $(35 \% \pm 5.4 \%$ vs $19 \% \pm 4.3 \%, P<.002)$, maximum pressure $(72 \pm 9.3$ vs $61 \pm 2.9$ $\mathrm{mm} \mathrm{Hg}, P<.05)$, and end-systolic pressure (67 \pm 7.0 vs $55 \pm 7.0 \mathrm{~mm} \mathrm{Hg}, P<.05)$. Enhanced myocardial preservation was demonstrated by enhanced left ventricular border zone wall thickness. Increased myocardial proliferation was evidenced by increased expression of proliferating cell nuclear antigen expression in cyclin A2-treated hearts.

Conclusions: In failing hearts, targeted delivery of cyclin A2 improves hemodynamic function, as measured by echocardiography and pressure catheter analysis, preserves ventricular wall thickness, and may serve as an ideal myocardial regenerative therapy.

$\mathrm{H}$ eart failure comprises a progressively prevalent global medical concern. Present medical and surgical therapies have variable efficacy, prompting investigation into molecular and cellular therapies to enhance myocardial repair. To date, cellular transplantation appears to show the greatest promise. However, this approach is hindered by multiple limitations relating to cell harvest, isolation, propagation, delivery, and survival as well as controversy stemming from the lack of clarity in defining the specific cell phenotype of greatest therapeutic benefit. This is most apparent in the fact that there are several studies that demonstrate functional improvement with the administration of unfractionated bone marrow, indicating a lack of specificity and ill-defined mechanism to this therapy. As an 


\author{
Abbreviations and Acronyms \\ cdk = cyclin-dependent kinase \\ LAD = left anterior descending coronary artery \\ LVIDs = left ventricular end-systolic diameter \\ LVIDd $=$ left ventricular end-diastolic diameter \\ OCT = optimal cutting temperature \\ PCNA = proliferating cell nuclear antigen
}

alternate approach, enhancing endogenous, preexistent myocardial reparative mechanisms has been investigated. One specific strategy to endogenously enhance functional myocardial tissue involves the induction of native cardiomyocyte cell cycle reentry and replication. ${ }^{1-4}$ Cyclins and cyclin-dependent kinases (cdks) are cell cycle regulators that present potential therapeutic targets. ${ }^{5}$ Overexpression of the restriction point control molecule cyclin D has yielded positive initial findings. ${ }^{6}$

Cyclin A2 is a unique cell cycle regulator that controls both the G1/S transition into DNA synthesis as well as the G2/M entry into mitosis as compared with cyclin D, which only regulates a single point in the cell cycle. This enhanced involvement in the cellular regenerative process makes cyclin A2 a particularly attractive therapeutic target. ${ }^{7}$ We have previously observed that transgenic mice constitutively expressing myocardial cyclin A2 develop postnatal cardiomyocyte mitosis and hyperplasia. ${ }^{8}$ There appears to be a $70 \%$ increase in the calculated number of cardiomyocytes. Subsequently, we have demonstrated that cardiac delivery of an adenoviral vector encoding cytomegalovirus promoterdriven cyclin A2 to rats with ischemic heart failure yields cyclin A2 transgene expression, initiates cardiomyocyte proliferation as measured by Ki-67 expression and bromodeoxyuridine uptake, stimulates cardiomyocyte mitosis as demonstrated by phosphohistone H3 expression, and increases myofilament density. ${ }^{9}$ In this study, the impact of this regenerative strategy on restoring ventricular function and geometry was investigated.

\section{Materials and Methods Animal Care}

The protocol outlining this study was approved by the University of Pennsylvania Institutional Animal Care and Use Committee. All animals received humane care in compliance with the "Guide for the Care and Use of Laboratory Animals" published by the US National Institutes of Health (NIH Publication No. 85-23).

\section{Adenoviral Vector Construction}

Recombinant replication-deficient (E1, E3 deleted) adenoviruses containing cyclin A2 driven by the cytomegalovirus promoter were produced and purified by the University of Iowa Gene Transfer Vector core. Empty, replication-deficient adenovirus containing no transgene was used as a control (Adeno Null, University of Iowa Gene Transfer Vector core). Direct intramyocardial injections of either adeno-cyclin A2 or adeno-null control virus were performed, and adenoviral transgene expression was confirmed by analysis of cyclin A2 protein levels by immunoblot and immunohistochemistry as previously described. ${ }^{9}$

\section{Animal Surgery}

Adult male Lewis inbred rats (250-300 g) were purchased from Charles River Laboratories (Boston, Mass). The rats were anesthetized with intraperitoneal ketamine $(75 \mathrm{mg} / \mathrm{kg})$ and xylazine $(7.5 \mathrm{mg} / \mathrm{kg})$, intubated with a 14-gauge angiocatheter, and mechanically ventilated with $1 \%$ isoflurane. A left fourth interspace thoracotomy was performed, and the proximal left anterior descending coronary artery (LAD) was ligated with a 7-0 polypropylene suture. Infarction was confirmed by visible blanching of the region at the time of ligation. As previously demonstrated, over a 6-week period untreated animals will predictably progress into congestive heart failure utilizing this model. ${ }^{10,11}$ This rodent experimental model will manifest active adverse ventricular remodeling during the early postinfarction period, which will then lead to left ventricular dilatation and contractile impairment. Immediately following LAD ligation, the animals were randomized to either treatment with adeno-cyclin A2 $(\mathrm{n}=8)$ or adeno-null $(\mathrm{n}=8)$ virus. The animals then received direct intramyocardial injections of $5 \times 10^{9}$ particle-forming units of virus into 5 separate locations in the peri-infarct border zone area. A total volume of $250 \mu \mathrm{L}$ was delivered, and injections were performed in a blinded fashion by a single investigator. Because adenoviral vector transgene expression will typically peak around 1 week after delivery and taper off by 3 to 4 weeks, the proliferative stimulus should be present during the period of most active adverse ventricular remodeling.

\section{Echocardiographic Evaluation of Left Ventricular Function}

Left ventricular function was evaluated 6 weeks after initial left anterior descending coronary artery ligation by transthoracic echocardiography utilizing a Phillips Sonos 5500 revD system (Phillips Medical Systems N.A., Bothwell, Wash) with a $12-\mathrm{MHz}$ transducer at an image depth of $3 \mathrm{~cm}$. Rats were anesthetized with intraperitoneal ketamine $(75 \mathrm{mg} / \mathrm{kg})$ and xylazine $(7.5 \mathrm{mg} / \mathrm{kg})$ and intubated with a 14-gauge angiocatheter and maintained on $1 \%$ isoflurane for the duration of echocardiographic assessment. Echocardiographic electrodes were placed on both the front limbs and right hind limb to obtain electrocardiographic tracings. Left parasternal and left apical echocardiographic measurements were obtained in the dorsal recumbency position. Two-dimensional left ventricular parasternal short-axis measurements were used to define internal diameters during systolic and diastolic time points. The left ventricular end-systolic diameter (LVIDs) and left ventricular end-diastolic diameter (LVIDd) were measured according to the American Society for Echocardiography leading-edge method from at least 3 consecutive cardiac cycles.

LVIDd and LVIDs were then used to later derive diastolic volume $\left(1.047 \times \mathrm{LVIDd}^{3}\right)$, systolic volume $\left(1.047 \times \mathrm{LVIDs}^{3}\right)$, fractional shortening ([(LVIDd-LVIDs/LVIDd)] $\times 100)$, stroke volume (diastolic volume-systolic volume), ejection fraction (stroke volume/diastolic volume $\times 100$ ), and cardiac output 


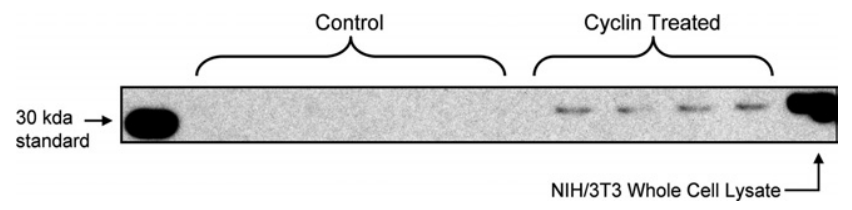

Figure 1. Immunoblot of PCNA showing cellular proliferation in cyclin-treated animals versus controls. PCNA, proliferating cell nuclear antigen.

(stroke volume $\times$ heart rate). M-mode echocardiography was used to define left ventricular diameters.

\section{Microtip Conductance Catheter Assessment of Left Ventricular Function}

Immediately following echocardiography, all rats underwent median sternotomy for assessment of left ventricular pressures. A $2 \mathrm{~F}$ pressure conductance microcatheter (Millar Instruments, Houston, Tex) was inserted into the left ventricle through the apex of the heart. Hemodynamic measurements were analyzed in a blinded fashion utilizing Chart v4.1.2 software (AD Instruments, Colorado Springs, Col) and ARIA1 Pressure Volume Analysis software (Millar Instruments).

\section{Quantitative Evaluation of Ventricular Geometry}

Prior to explantation, hearts were perfused and rinsed with 10 $\mathrm{mL}$ phosphate-buffered saline solution via the aortic root until visual confirmation of blanching of the myocardium. The hearts were then explanted, and the left ventricular cavity was filled with optimal cutting temperature (OCT) embedding compound fixative through the aortic root at a fixed distending pressure. The distended heart was placed in OCT, snap-frozen in liquid nitrogen, and stored in a $-80^{\circ} \mathrm{C}$ freezer. Measurements of ventricular geometry were performed on $10 \mu \mathrm{m}$-thick slices at the level of the midpapillary muscle.
Hematoxylin and eosin stain was performed to assess the potential therapeutic effects of cyclin A2 on preserving ventricular geometry. The ventricular border zone wall thickness was measured in 2 distinct sections and averaged. Measurements were performed on digitized photomicrographs using Scion Image processing software (Scion Corp, Frederick, Md) with standards of known millimeter length. For all analyses, the border zone was defined as the viable myocardial tissue immediately adjacent to the infarct scar. All morphometric studies were performed by 2 examiners who were blinded to treatment. Additionally, to gauge the severity of myocardial fibrosis, Masson trichrome staining (Accustain Trichrome Stain, Sigma, St Louis, Mo) was also performed on frozen sections from each tissue block. Collagen in the tissue sections was stained blue using this trichrome method and qualitatively compared.

\section{Nuclear Proliferation as Assessed by Immunoblot and Immunofluorescence}

To determine DNA synthesis and proliferation, immunoblotting for proliferating cell nuclear antigen (PCNA) was performed. Myocardial tissue obtained from the peri-infarct border zone was homogenized in buffer consisting of $50 \mathrm{mM}$ tris/ $\mathrm{HCl}(\mathrm{pH} 7.5), 100$ $\mathrm{mM} \mathrm{NaCl}, 5 \mathrm{mM}$ ethylenediamine-tetraacetic acid, $1 \% \mathrm{v} / \mathrm{v}$ Triton $\mathrm{X}-100,1 \mathrm{mM} \mathrm{NaF}, 1 \mathrm{mM} \mathrm{Na} \mathrm{VO}_{4}, 0.2 \mathrm{mM}$ phenymethylsulfonyl fluoride, $10 \mu \mathrm{g} / \mathrm{mL}$ leupeptin, and $10 \mu \mathrm{g} / \mathrm{mL}$ aprotinin. Lysates were cleared by centrifugation at $12,000 \mathrm{~g}$ for 10 minutes at $4^{\circ} \mathrm{C}$ and analyzed for protein content via the Bradford method (BioRad, Hercules, Calif). Forty micrograms of each protein sample were then denatured at $70^{\circ} \mathrm{C}$ for 10 minutes and electrophoresed onto a $4 \%$ to $12 \%$ gradient sodium dodecyl sulfate-polyacrylamide gel. Equal loading of protein was verified by Coomassie blue staining. Proteins were transferred to Immobilon-P polyvinylidane fluoride membranes (Millipore, Bedford, Mass), and immunoblotting was performed using a rabbit anti-rat PCNA polyclonal antibody (Santa Cruz Biotechnology, Santa Cruz, Calif). Proteins were visualized via horseradish peroxidase-conjugated anti-rabbit antibody (Amersham Biosciences, Piscataway, NJ) and chemiluminescence detection (Amersham Biosciences). Immuno-
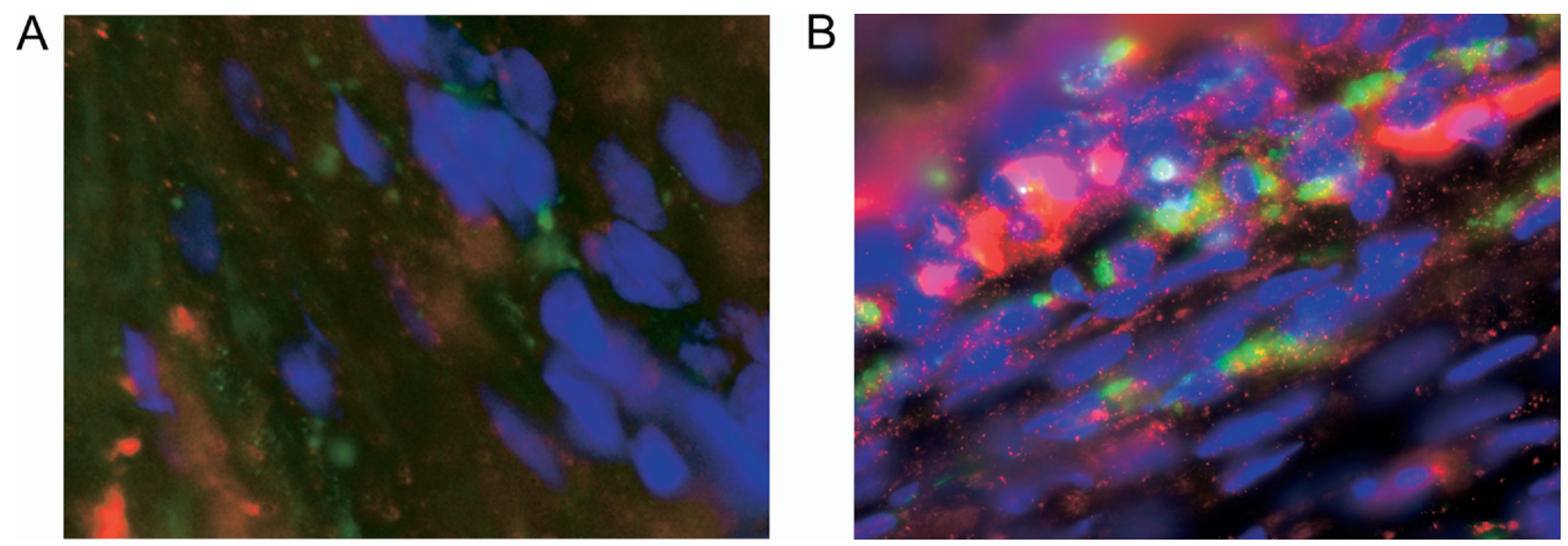

Figure 2. Immunofluorescent colocalization of PCNA and $\alpha$-sarcomeric actin expression in null hearts (A) and in cyclin-treated hearts (B), demonstrating cardiomyocyte proliferation. PCNA, proliferating cell nuclear antigen. 
TABLE 1. Hemodynamic assessment of left ventricular function

\begin{tabular}{lccc}
\hline & $\begin{array}{c}\text { Null } \\
(\mathbf{n = 8 )}\end{array}$ & $\begin{array}{c}\text { Cyclin } \\
(\mathbf{n = 8 )}\end{array}$ & $\begin{array}{c}\boldsymbol{P} \\
\text { value }\end{array}$ \\
\hline Heart rate (bpm) & $242 \pm 24$ & $215 \pm 35$ & $\mathrm{NS}$ \\
Ejection fraction (\%) & $46 \pm 8.5$ & $72 \pm 7.4$ & $<.002$ \\
Fractional shortening (\%) & $19 \pm 4.3$ & $35 \pm 5.4$ & $<.002$ \\
LV internal diameter systole (mm) & $5 \pm 0.5$ & $3.9 \pm 0.7$ & $<.05$ \\
LV end systolic volume (mm ${ }^{3}$ ) & $130 \pm 40$ & $70 \pm 40$ & $<.05$ \\
Stroke volume (mL) & $0.11 \pm 0.04$ & $0.16 \pm .04$ & $<.05$ \\
Cardiac output (mL/min) & $26 \pm 5.0$ & $32 \pm 3.3$ & $<.05$ \\
LV wall thickness (mm) & $1.9 \pm 0.3$ & $2.6 \pm 0.4$ & $<.05$ \\
Maximum pressure (mm Hg) & $61 \pm 2.9$ & $72 \pm 9.3$ & $<.05$ \\
End-systolic pressure (mm Hg) & $55 \pm 7.0$ & $67 \pm 7.0$ & $<.05$
\end{tabular}

bpm, Beats per minute; $L V$, left ventricular.

histochemical imaging of PCNA was performed on myocardial thin sections $(10 \mu \mathrm{m})$ fixed in $4 \%$ paraformaldehyde, permeabilized with Triton X-100, and blocked with 5\% normal goat serum. Proliferating cardiomyocytes were colocalized with
PCNA (1:600 rabbit anti-human PCNA, Abcam Cambridge, Mass) and $\alpha$-sarcomeric actin (1:700 mouse anti-rabbit $\alpha$-sarcomeric actin, Sigma Aldrich USA [St. Louis, MO]) as well as 4',6diamidino-2-phenylindole and imaged $(40 \times$ air magnification, Leica Microsystems, Wetzlar, Germany).

\section{Statistical Analysis}

Quantitative data are expressed as means \pm standard error of the mean. Statistical significance was evaluated using the unpaired Student $t$ test for comparison between 2 means.

\section{Results}

\section{Expression of Cyclin A2 Enhances Myocardial} Proliferation

Immunoblotting via Western blot revealed higher levels of PCNA in the border zone of cyclin-treated hearts versus null controls. There was no visible expression of PCNA in the control group, whereas a significant expression of PCNA was evidenced in the cyclin-A2-treated group (Figure 1). Enhanced expression of PCNA signified enhanced nuclear

\section{A}

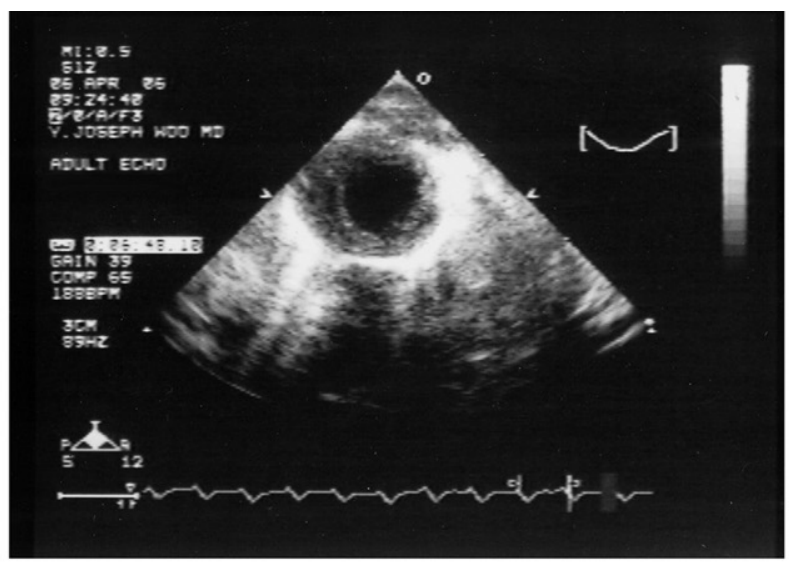

B

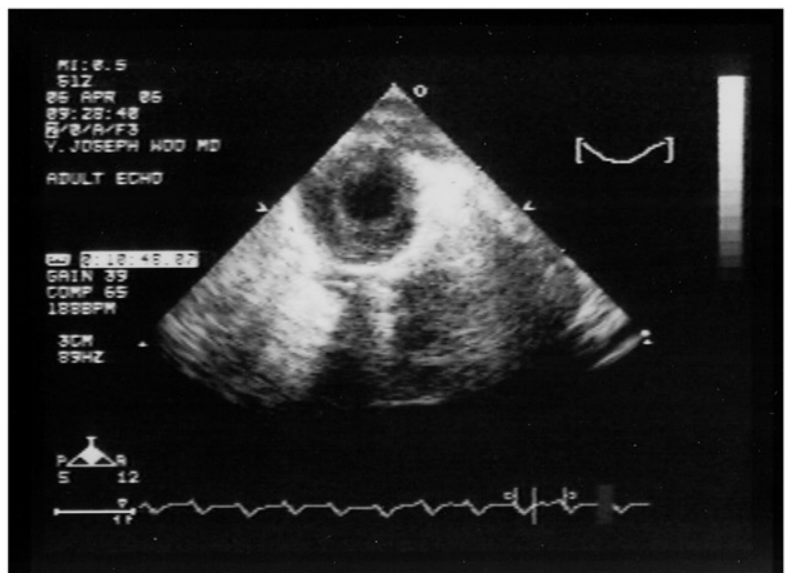

C

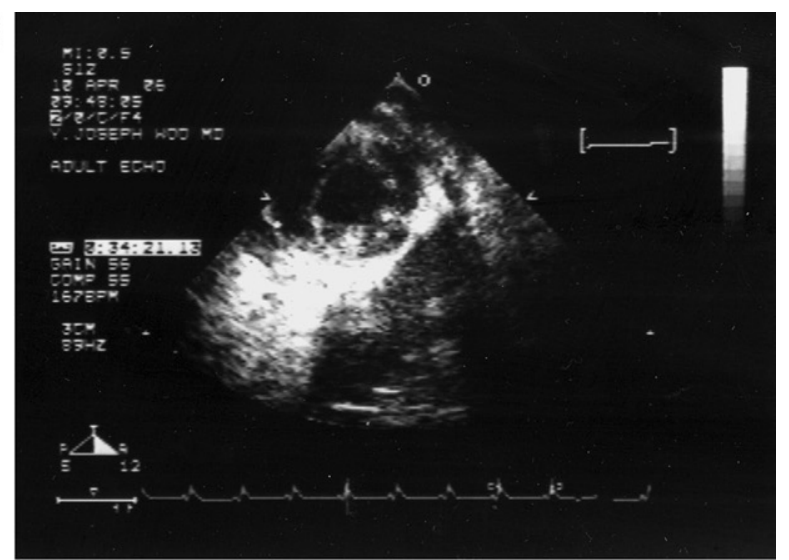

$\mathrm{D}$

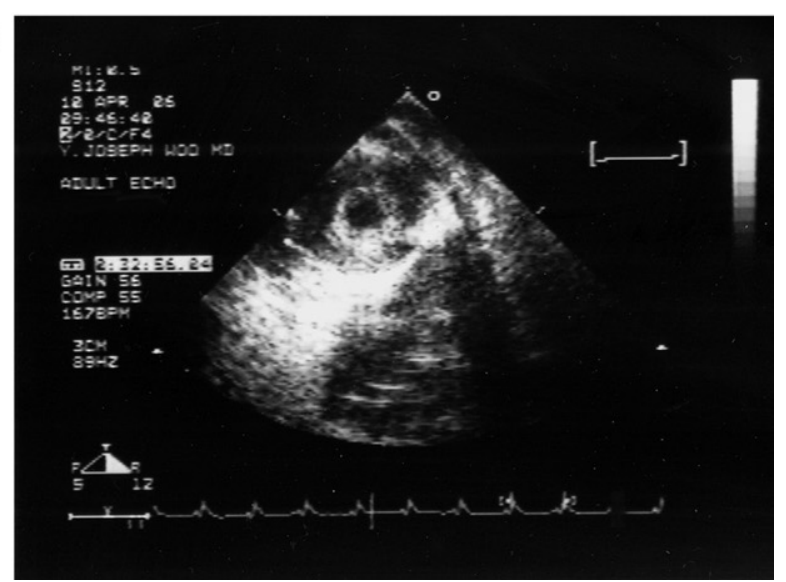

Figure 3. Echocardiographic functional evaluation of hearts 6 weeks postligation. Representative 2-D parasternal short-axis views of a control heart and a cyclin-treated heart. A, Control heart in diastole. B, Control heart in systole. C, Cyclin-treated heart in diastole. D, Cyclin-treated heart in systole. Cyclin-treated hearts demonstrate enhanced ejection fraction as compared with control hearts. 

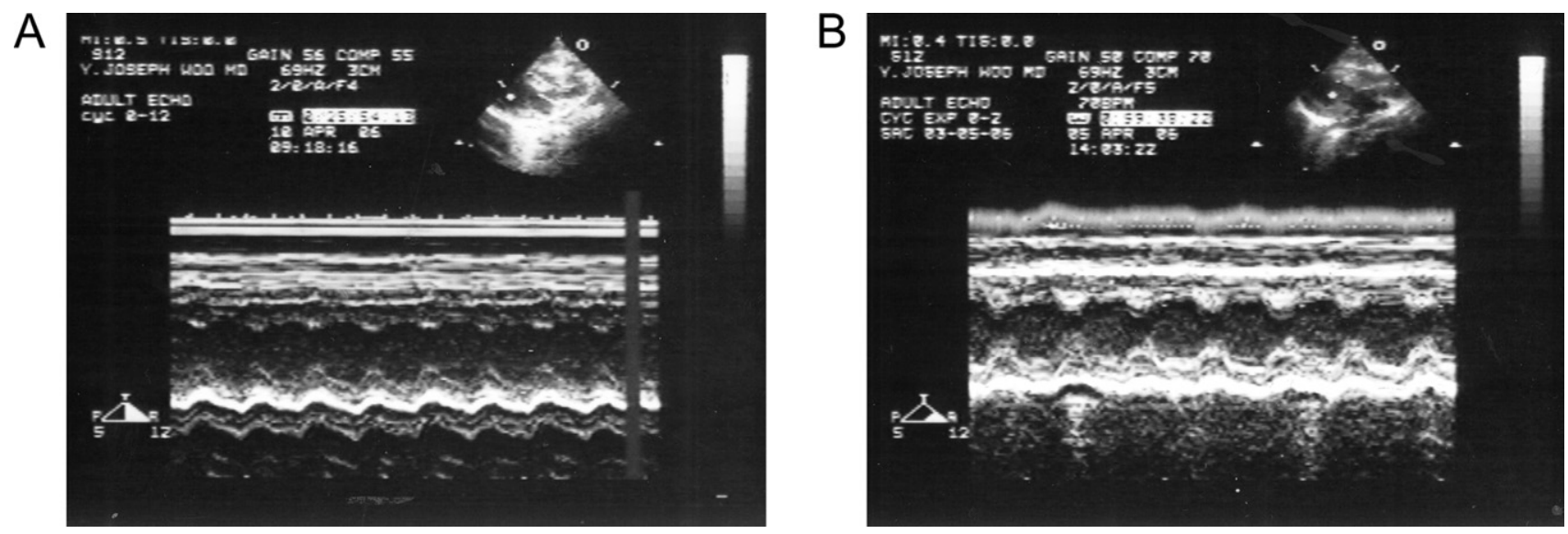

Figure 4. Representative parasternal long-axis M-mode view of a control heart (A) and cyclin-treated heart (B) showing increased wall thickening in the cyclin-treated heart.

proliferation in the treatment group as compared with control. Qualitative immunofluorescent colocalization of PCNA and $\alpha$-sarcomeric actin was observed in cyclin-treated animals (Figure 2).

\section{Cyclin A2 Therapy Enhances Myocardial Function}

Echocardiographic analysis performed 6 weeks after ligation showed statistically significant improvements in hemodynamic function as evidenced by left ventricular systolic volume, fractional shortening, ejection fraction, stroke volume, and cardiac output (Table 1). Parasternal short-axis echocardiographic images demonstrate a significantly higher ejection fraction in cyclin-treated hearts versus controls (Figure 3). Left ventricular wall thicknesses were obtained from the parasternal long-axis M-mode view at the level of the papillary muscle and were significantly thicker in the cyclin-treated group (Table 1). Representative parasternal long-axis M-mode echocardiographic images from cyclin-treated and control hearts revealed significantly preserved left ventricular end-systolic internal diameter in cyclin-treated hearts versus controls (Figure 4). In vivo pressure analysis by Millar catheter following echocardiographic analysis yielded statistically significantly higher maximum pressure and end-systolic pressure in cyclin-treated animals versus null controls (Table 1).

Cyclin A2 Therapy Enhances Myocardial Preservation Morphometric analysis for border zone ventricular thickness showed significantly preserved left ventricular wall thickness in cyclin-treated hearts as compared with controls $(1.7 \pm 0.06$ vs $1.5 \pm 0.06 \mathrm{~mm}, P<.05$; Figure 5$)$. Collagen staining by Masson's trichrome method revealed increased viable myocardial tissue and a diminished scar fraction in the cyclin-treated group when compared with null control (Figure 5). This was further seen as a reduction in collagen deposition in the border zone of cyclin-treated tissue specimens.

\section{Discussion}

Cell cycle control entails a complex network of cyclins, cdks, cdk inhibitors, and multiple additional regulators. Because of their central role in this control, the cyclins serve as attractive potential therapeutic targets to stimulate endogenous cardiomyocyte replication as a myocardial regeneration and repair strategy. Cyclin A2 possesses several unique features that render it a particularly appealing therapeutic target. It controls both major cell cycle transition points G1/S and G2/M. It is the only cyclin that is completely silenced after birth in mice, rats, and humans. ${ }^{8,12}$ The rate of cyclin A2 decrement matches the rate of cardiomyocyte cell cycle withdrawl. ${ }^{11}$ Alternative targets proposed include the $\mathrm{D}$ and E family cyclins. However, the majority of mouse tissues develop without all 3 D-type cyclins. ${ }^{13}$ Cyclin E1 and E2 knockout mice develop normally. ${ }^{14}$ On the other hand, a cyclin A2 knockout is embryonically lethal at embryonic day $5.5 .^{15}$

Thus we have focused upon the alteration of cyclin A2 as a potential heart failure treatment strategy. In a transgenic cyclin A2 mouse, we have previously reported cardiomyocyte mitosis and hyperplasia with up to a $70 \%$ increase in the number of cardiomyocytes. ${ }^{8}$ With a postinfarction, therapeutic adenoviral delivery model, we have observed robust cyclin A2 transgene expression, cardiomyocyte proliferation and mitosis, and enhanced cardiac myofibril density. ${ }^{9}$ This particular model has been designed to yield high-level transient cyclin A2 expression during a 2- to 4-week period after myocardial infarction, during which significant adverse remodeling stimuli are in effect. This limited time 

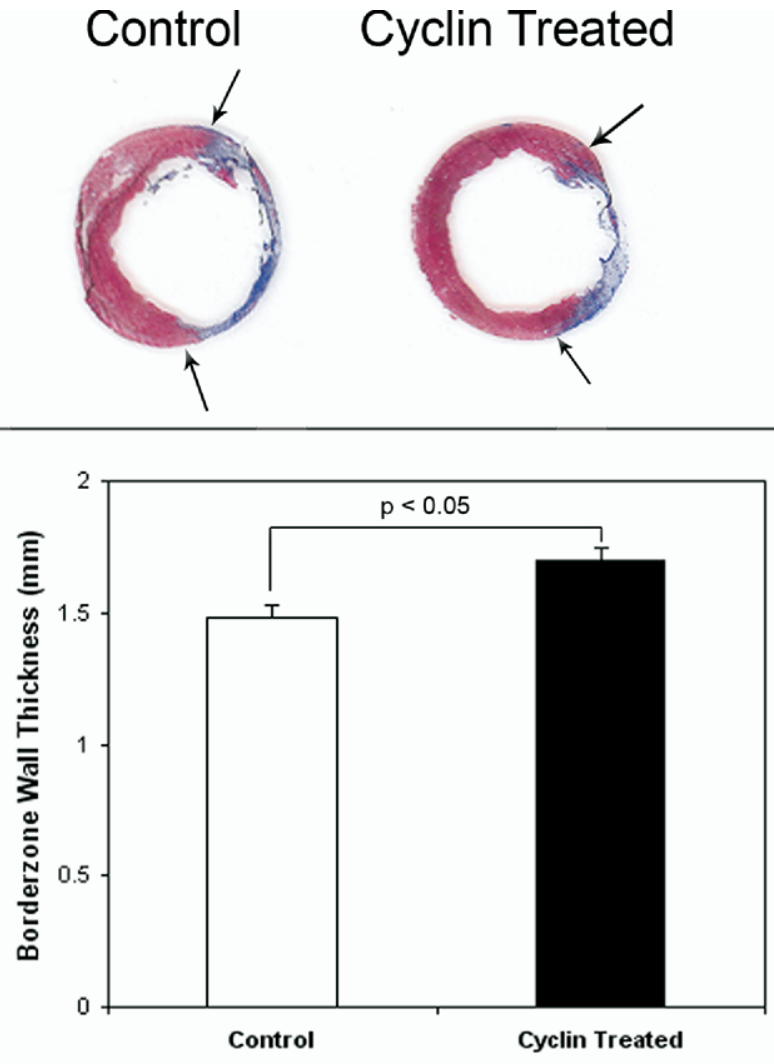

Figure 5. Representative tissue sections stained by the Masson's trichrome method show qualitatively less fibrosis (areas stained blue) in cyclin-treated hearts versus control hearts. Planimetry measurements of the ventricular wall thickness at border zone (arrows) show more-preserved wall thickness in cyclin-treated animals versus controls.

frame of transgene expression obviates any potential detrimental effects of uncontrolled, induced myocardial hyperplasia. This should also prevent any theoretical risk of neoplasia. In fact, in the transgenic cyclin A2-treated animals examined at age 1.5 years, despite constitutive myocardial cyclin A2 expression, there was no histologic evidence of neoplasia. ${ }^{8}$ The use of an adenoviral gene therapy system can be criticized for its inherent limited ultimate human clinical translatability. However, as a research model, it is highly effective in providing high-level, targeted expression of a gene of interest. An additional limitation of this approach, although theoretical, is the potential need for a nuclear localization signal on an adenoviral transgene designed to affect a nuclear process. Although this has been postulated, ${ }^{16}$ there are multiple other studies that have demonstrated that adenoviral transgene products can be properly posttranslationally modified and targeted to the nucleus., ${ }^{517-19}$ Another possible limitation is that mechanistically, cyclin ex- pression did not induce cardiomyocyte proliferation but rather cardiomyocyte preservation through antiapoptotic or other cell survival pathways. In light of the extensive cardiomyocyte proliferative and mitotic evidence observed in this and prior experiments, this seems less likely.

We have previously reported an extensive molecular and cellular analysis of cardiomyocyte proliferation, mitosis, and regeneration. ${ }^{9}$ Thus for the current study, we selected only 1 proliferation marker to serve as an internal measure of consistency between studies. The results with PCNA reveal active myocardial proliferation within the cyclin A2treated animals. To examine the functional and geometric benefits of myocardial regeneration, we elected to conduct closed chest hemodynamic studies with transthoracic echocardiography and then add corroborative data with intraventricular pressure monitoring. The echocardiography data reveal a uniform improvement in multiple myocardial functional parameters in the cyclin group compared with controls. Echocardiography provides an advantage over other functional assays in that it provides images and data that are conceptually clinically pertinent. The histologic assays complement the echocardiographic data. The improvement in ventricular geometry is displayed with both the scar analysis and the wall thickness assessment. These findings are consistent with the induction of a myocardial reparative mechanism.

\section{Conclusions}

Therapeutic expression of cyclin A2 in infarcted hearts induced proliferation and yielded echocardiographic and intracavitary pressure evidence of enhanced cardiac function as well as improved ventricular geometry. This cell cycle regulator presents an attractive therapeutic target for the induction of myocardial regeneration as a treatment for heart failure.

\section{References}

1. Bicknell KA, Coxon CH, Brooks G. Forced expression of the cyclin B1-CDC2 complex induces proliferation in adult rat cardiomyocytes. Biochem J. 2004;382(Pt 2):411-6.

2. Regula KM, Rzeszutek MJ, Baetz D, et al. Therapeutic opportunities for cell cycle re-entry and cardiac regeneration. Cardiovasc Res. 2004;64:395-401.

3. Regula KM, Kirshenbaum LA. Breaking down cell-cycle barriers in the adult heart. Circ Res. 2004;94:1524-6.

4. Soonpaa MH, Field LJ. Survey of studies examining mammalian cardiomyocyte DNA synthesis. Circ Res. 1998;83:15-26.

5. Brooks G, Poolman RA, McGill CJ, et al. Expression and activities of cyclins and cyclin-dependent kinases in developing rat ventricular myocytes. J Mol Cell Cardiol. 1997;29:2261-71.

6. Pasumarthi KB, Nakajima H, Nakajima HO, Soonpaa MH, Field LJ. Targeted expression of cyclin D2 results in cardiomyocyte DNA synthesis and infarct regression in transgenic mice. Circ Res. 2005; 96:110-8.

7. Li JM, Poolman RA, Brooks G. Role of G1 phase cyclins and cyclindependent kinases during cardiomyocyte hypertrophic growth in rats. Am J Physiol. 1998;275:H814-22.

8. Chaudhry HW, Dashoush NH, Tang H, et al. Cyclin A2 mediates cardiomyocyte mitosis in the postmitotic myocardium. J Biol Chem. 2004;279:35858-66. 
9. Woo YJ, Panlilio CM, Cheng RK, Liao GP, Atluri P, Cohen JE, et al. Therapeutic delivery of cyclin A2 induces myocardial regeneration and enhances cardiac function in ischemic heart failure. Circulation. 2006;114:206-13.

10. Liu YH, Yang XP, Nass O, Sabbah HB, Peterson E, Carretero OA. Chronic heart failure induced coronary artery ligation in Lewis inbred rats. Am J Physiol. 1997;272:H722-7.

11. Woo YJ, Grand TJ, Berry MF, Atluri P, Moise MA, Hsu VM, et al. Stromal cell-derived factor and granulocyte-monocyte colonystimulating factor form a combined neovasculogenic therapy for ischemic cardiomyopathy. J Thorac Cardiovasc Surg. 2005;130:321-9.

12. Yoshizumi M, Lee WS, Hsieh CM, Tsai JC, Li J, Perrella MA, et al. Disappearance of cyclin A correlates with permanent withdrawal of cardiomyocytes from the cell cycle in human and rat hearts. $J$ Clin Invest. 1995;95:2275-80.

13. Kozar K, Ciemerych MA, Rebel VI, Shigematsu H, Zagozdzon A, Sicinska E, et al. Mouse development and cell proliferation in the absence of D-cyclins. Cell. 2004;118:477-91.

14. Geng Y, Yu Q, Sicinska E, Das M, Schneider JE, Bhattacharya S, et al. Cyclin E ablation in the mouse. Cell. 2003;114:431-43.

15. Murphy M, Stinnakre MG, Senamaud-Beaufort C, Winston NJ, Sweeney C, Kubelka M, et al. Delayed early embryonic lethality following disruption of the murine cyclin A2 gene. Nat Genet. 1999; 23:481-6.

16. Tamamori-Adachi M, Ito H, Sumrejkanchanakij P, Adachi S, Hiroe M, Shimizu M, et al. Critical role of cyclin D1 nuclear import in cardiomyocyte proliferation. Circ Res. 2003;92:e12-9.

17. Agah R, Kirshenbaum LA, Abdellatif M, et al. Adenoviral delivery of E2F-1 directs cell cycle reentry and p53-independent apoptosis in postmitotic adult myocardium in vivo. J Clin Invest. 1997;100: 2722-8.

18. Akli S, Zhan S, Abdellatif M, Shneider MD. E1A can provoke G1 exit that is refractory to p21 and independent of activating cdk2. Circ Res. 1999;85:319-28.

19. Suzuki G, Lee TC, Fallavollita JA, Canty JM Jr. Adenoviral gene transfer of FGF-5 to hibernating myocardium improves function and stimulates myocytes to hypertrophy and reenter the cell cycle. Circ Res. 2005;96:767-75.

\section{Discussion}

Dr Frank W. Sellke (Boston, MA). Joe, This is a very nice extension of what was presented at the American Heart Associa- tion last year. It suggests that cyclin A2 may increase myocardial regeneration and may have some therapeutic effect in treatment of patients with chronic heart failure. I do have a couple of questions. How did the cyclin A2 affect other cell cycle proteins and factors regulating myocardial regeneration and function? You may have looked at this previously, but do you have data?

Dr Y. Joseph Woo (Philadelphia, Pa). There is work done in the transgenic mouse that constitutively expressed cyclin A2. There did not appear to be much change in the other cell cycle regulators. It seems that cyclin A was working independently of manipulation of the other factors in the cell cycle regulation pathway.

Dr Sellke. The other question I have is a follow-up to that question. How does manipulation of cyclin A2 affect long-term viability? Could it just be a short-term effect with aggression of these new cells at a later date?

Dr Woo. Yes. Cyclin D has been evaluated in a similar fashion by others. And although there is some controversy regarding whether that is actually effective, similar results have been shown. Cyclin D is notable for its control of the restriction point, that is, the G1, theoretical G0 point interaction. Cyclin A is particularly interesting, primarily for the points that I mentioned earlier. It is a 2-point controller. It drives G1 into DNA synthesis. There are various factors that can drive proliferation but not actually replication. You need to actually be able to push a cell into mitosis to make a difference. Cyclin A also regulates this control point. Furthermore, a cyclin A2 knockout is an embryonic lethal variant and also, it is totally silenced in the adult, thus making it an appealing therapeutic target.

Dr Sellke. Have you looked at targeting 2-cell cycle regulator factors simultaneously?

Dr Woo. That is a good idea. I think the thing to do here would be to use cyclins D and A. Cyclin D would allow the cells to come out of the theoretical G0 state, and then cyclin A would push the cells into $\mathrm{S}$ and then over into $\mathrm{M}$ state. 